Environnement urbain

Urban Environment

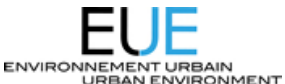

\title{
Analyse de cycle de vie à l'échelle du quartier : un outil d'aide à la décision? Le cas de la ZAC Claude Bernard à Paris (France)
}

\author{
Morgane Colombert, Cédissia de Chastenet, Youssef Diab, Christophe Gobin, \\ Grégory Herfray, Thibaut Jarrin, Bruno Peuportier, Charlotte Tardieu et \\ Maxime Trocmé
}

Volume 5, 2011

URI : https://id.erudit.org/iderudit/1007605ar

DOI : https://doi.org/10.7202/1007605ar

Aller au sommaire du numéro

Éditeur(s)

Réseau Villes Régions Monde

ISSN

1916-4645 (numérique)

Découvrir la revue

Citer cet article

Colombert, M., de Chastenet, C., Diab, Y., Gobin, C., Herfray, G., Jarrin, T., Peuportier, B., Tardieu, C. \& Trocmé, M. (2011). Analyse de cycle de vie à l'échelle du quartier : un outil d'aide à la décision? Le cas de la ZAC Claude Bernard à Paris (France). Environnement urbain / Urban Environment, 5, c1-c21. https://doi.org/10.7202/1007605ar
Résumé de l'article

Les collectivités territoriales s'engagent dans de nombreuses actions pour limiter leurs impacts environnementaux. La ville de Paris cherche à se doter de nouveaux outils permettant de mesurer l'incidence de choix urbains ou architecturaux sur l'environnement dès la phase de conception. Les outils d'analyse du cycle de vie (ACV) permettent aujourd'hui d'évaluer les performances tout au long de la vie d'un ouvrage : construction, exploitation, fin de vie. La recherche présentée dans cet article a permis d'effectuer l'ACV de la ZAC Claude Bernard à Paris et d'observer le potentiel d'aide à la décision pour la maitrise d'ouvrage d'un tel outil. 


\title{
ANALYSE DE CYCLE DE VIE À L'ÉCHELLE DU QUARTIER : UN OUTIL D'AIDE À LA DÉCISION? LE CAS DE LA ZAC CLAUDE BERNARD À PARIS (FRANCE)
}

\author{
Morgane Colombert, Cédissia de Chastenet, \\ Youssef DiaB, Christophe Gobin, Grégory Herfray, \\ Thibaut JARRIN, Bruno PEUPORTIER, \\ Charlotte TARDIEU et Maxime TrocmÉ
}

\begin{abstract}
I RÉSUMÉ
Les collectivités territoriales s'engagent dans de nombreuses actions pour limiter leurs impacts environnementaux. La ville de Paris cherche à se doter de nouveaux outils permettant de mesurer l'incidence de choix urbains ou architecturaux sur l'environnement dès la phase de conception. Les outils d'analyse du cycle de vie (ACV) permettent aujourd'hui d'évaluer les performances tout au long de la vie d'un ouvrage : construction, exploitation, fin de vie. La recherche présentée dans cet article a permis d'effectuer l'ACV de la ZAC Claude Bernard à Paris et d'observer le potentiel d'aide à la décision pour la maitrise d'ourrage d'un tel outil.
\end{abstract}

MOTS-CLÉS - Analyse de cycle de vie, projet d'aménagement urbain, outil d'aide à la décision, qualité environnementale, étude comparative

\begin{abstract}
- ABSTRACT
Local authorities are engaged in numerous actions in favor of the environment. The City of Paris is interested in new tools to take into account environmental impacts of their urban planning projects during the different stages of the project design. Life Cycle Assessment (LCA) tools allow environmental impacts of a building to be evaluated during its life: construction, exploitation, end of life. In this article, we test an LCA tool on an urban planning project in Paris (France) in order to analyze the relevance of LCA for assessing district impacts and being a decision support tool.
\end{abstract}

KEYWORDS - Life cycle assessment, urban planning project, decision support tool, environmental quality, comparative study

1 Coordonnées des auteurs : Morgane Colombert, morgane.colombert@eivp-paris.fr; Cédissia de Chastenet, cedissia.dechastenet@paris.fr; Youssef Diab, youssef.diab@eivp-paris.fr; Christophe Gobin, christophe.gobin@vinci-construction.fr; Grégory Herfray, gregory.herfray@mines-paristech.fr; Thibaut Jarrin, thibaut.jarrin@gmail.com; Bruno Peuportier, bruno.peuportier@mines-paristech.fr; Charlotte Tardieu, charlotte.tardieu@eivp-paris.fr; Maxime Trocmé, maxime.trocme@vinci-construction.fr 


\section{INTRODUCTION}

Ville zéro carbone, ville sans $\mathrm{CO}_{2}$, ville postcarbone, ville neutre en carbone; à l'heure des changements climatiques mondiaux, la course vers une efficacité énergétique toujours plus grande, vers une réduction des besoins en énergie et vers une production non émettrice de gaz à effet de serre est lancée dans un grand nombre de villes. II est vrai que celles-ci constituent un enjeu majeur dans la lutte contre les changements climatiques car elles représentent plus des deux tiers de la consommation mondiale d'énergie, plus de $70 \%$ des rejets en gaz à effet de serre mais également qu'elles accueillent plus de $50 \%$ de la population mondiale.

La Ville de Paris, sensible aux enjeux climatiques mondiaux, a adopté son Plan Climat territorial en 2007 et s'est engagée, pour 2020, à réduire de $25 \%$ les émissions de gaz à effet de serre et les consommations énergétiques de son territoire, et à atteindre une proportion de $25 \%$ de consommation énergétique du territoire provenant d'énergies renouvelables. Sur ses compétences propres, la Ville de Paris, souhaitant être elle-même exemplaire, a monté ce pourcentage à $30 \%$.

L'énergie n'est pas la seule problématique environnementale pour laquelle la Ville de Paris s'engage et parmi les nombreuses actions mises en œuvre, nous pouvons citer: le Plan Biodiversité, le référentiel "Un aménagement durable pour Paris », les diagnostics de performance énergétique des équipements de la ville, les labellisations « éco-jardin » des espaces verts, etc.

Pour atteindre ces objectifs pluriels, la ville de Paris cherche à se doter de nouveaux outils permettant de mesurer l'incidence de choix urbains ou architecturaux sur l'environnement (énergie, polluants atmosphériques, déchets, etc.), et ce dès la phase de conception.

La Ville de Paris a développé depuis 2007 un référentiel intitulé «Un aménagement durable pour Paris ». Ce référentiel fixe, d'une part, des objectifs qualitatifs et quantitatifs et, d'autre part, propose aux acteurs de l'urbanisme un tableau de bord et des indicateurs d'évaluation et de suivi des opérations d'aménagement.

II ne s'agit plus seulement d'évaluer un projet finalisé, il s'agit désormais de développer des outils permettant d'identifier dès le départ les impacts prévisibles du projet sur l'environnement. Ces outils devront ainsi permettre d'apporter une aide à la conception pour les maitres d'œurre, et ce, de la naissance du projet à sa mise en œuvre, mais également d'aiguiller les maitres d'ouvrage dans leurs décisions.

Les outils d'analyse du cycle de vie (ACV), initialement conçus pour les produits du secteur industriel, sont aujourd'hui de plus en plus utilisés pour évaluer les performances environnementales du secteur de la construction (Chevalier, 2009). Ils permettent d'évaluer les performances tout au long de la vie d'un ouvrage : construction, exploitation et fin de vie (démolition et éventuellement recyclage). Cependant, sont-ils véritablement adaptés pour l'aide à la décision des collectivités territoriales? Sont-ils réellement en accord avec les différents temps d'un projet d'aménagement? Permettent-ils d'aider le décideur à analyser les impacts environnementaux de son projet? Permettent-ils de guider, en phases initiale et finale, les décisions sur le projet?

La recherche présentée dans cet article résulte d'un partenariat entre la Direction de l'Urbanisme de la Ville de Paris, le Centre Énergétique et Procédés des Mines Paristech, Vinci Construction France et l'École des Ingénieurs de la Ville de Paris. Elle a permis d'effectuer une première $A C V$ de la Zone d'Aménagement Concerté (ZAC) Claude Bernard à Paris et d'observer le potentiel d'aide à la décision d'un tel outil pour un projet d'aménagement, notamment en phase d'élaboration du plan masse.

\section{L'ANALYSE DE CYCLE DE VIE : DU BÂTIMENT À L'ÉCHELLE QUARTIER}

L'Analyse de cycle de vie consiste à quantifier les impacts environnementaux d'un produit du « berceau à la tombe », c'est-à-dire de l'extraction des matières premières à l'élimination des déchets. La norme ISO I 4040 spécifie les principes et le cadre applicables à la réalisation d'analyses du cycle de vie. Une étude ACV se déroule ainsi en quatre phases :

I) La définition des objectifs et du champ de l'étude : systèmes à étudier, fonctions de ces systèmes, unité fonctionnelle, frontières des systèmes étudiés, règles d'affectation, hypothèses, exigences sur les données de l'inventaire, limites du modèle, ou encore catégories d'impacts pris en compte.

2) L'inventaire des flux de matières et d'énergies entrant et sortant à chaque étape du cycle de vie du produit. Sont ainsi mesurés: les flux de matière entrant (matières premières, matériaux et consommables), les flux de matière sortant (coproduits, sous-produits, déchets liquides et solides, 


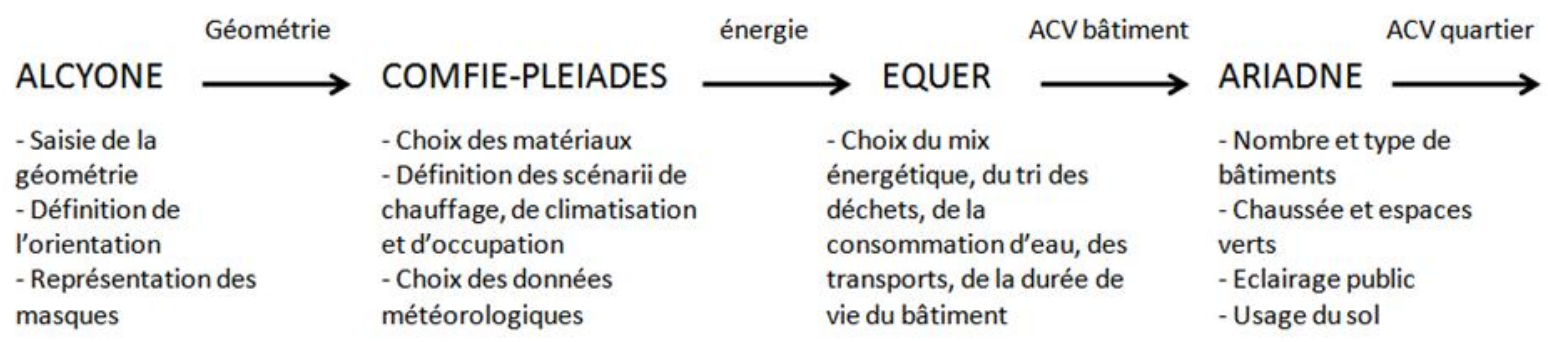

Fig. I - Chainage des entrées/sorties entre les outils d'évaluation

rejets dans l'air, dans l'eau et dans les sols) et les flux d'énergie entrant et sortant: énergie électrique, énergie thermique des différents combustibles, chaleur.

3) L'évaluation des impacts potentiels à partir des flux de matière et d'énergie recensés: cette phase d'analyse des impacts a pour objectif de mieux comprendre la signification environnementale de l'inventaire. On parle d'impacts potentiels en raison des nombreuses incertitudes, car les effets de synergie ou d'antagonisme entre polluants ou certaines caractéristiques locales ne sont pas pris en compte.

4) L'interprétation des résultats obtenus en fonction des objectifs fixés : l'interprétation se fait à chaque étape de manière à vérifier que l'on répond continuellement aux objectifs retenus. S'il n'est pas possible de répondre aux objectifs, ceux-ci doivent être redéfinis dans le cadre de la première étape de l'ACV. En phase finale de l'ACV, l'interprétation résume et analyse les résultats de l'étude afin de dresser des conclusions utiles à la prise de décision dans le cadre des objectifs fixés initialement. II est à noter que l'ACV est généralement utilisée pour proposer des améliorations au produit étudié.

Initialement développée pour les produits industriels, cette méthode est aujourd'hui utilisée dans le secteur de la construction à deux échelles (Chevalier, 2009): I) les matériaux et produits de construction; 2) les bâtiments ou ouvrages de construction. Plusieurs logiciels existent aujourd'hui pour faciliter ces analyses.

À l'échelle du bâtiment, plusieurs outils ont été développés dans le monde (Peuportier et al., 2004). Ces travaux ont commencé dès les années 1980 dans des pays comme la Suisse, l'Allemagne et les Pays Bas, et des ateliers de travail ont été organisés au début des années 1990, par exemple (Cole, 1992). Le projet européen REGENER a permis d'esquisser une première base méthodologique commune (Peuportier,
1997). D'autres outils du même type ont ensuite été développés en Grande Bretagne, en Finlande, en Autriche, au Canada, en Australie puis aux USA, etc. Parmi ceux développés en France, nous pouvons citer :

- EQUER : il a été développé par le centre énergétique et procédés de l'École des Mines Paristech (Polster, 1995). EQUER est associé à PLEIADES + COMFIE, un logiciel de simulation thermique, et il permet de calculer onze paramètres environnementaux en fonction des résultats de la simulation des consommations énergétiques en exploitation.

- TEAM ${ }^{\mathrm{TM}}$ Bâtiment : il a été développé par la société Ecobilan (Groupe PriceWaterhouse Coopers). II permet de modéliser et de comparer des bâtiments ou des quartiers et d'obtenir instantanément leur bilan environnemental multicritères (consommation de ressources, d'énergie et d'eau, génération de déchets, pollution de l'eau et de l'air, réchauffement climatique, etc.).

- ELODIE : c'est un outil de calcul de la performance environnementale des bâtiments développé par le Centre Scientifique et Technique du Bâtiment (CSTB) avec le concours de la Direction générale de l'urbanisme, de l'habitat et de la construction. Depuis 2010, ELODIE permet de faire des calculs d'énergie grise, le bilan carbone ou de calculer un profil environnemental multicritère d'un bâtiment pour faciliter l'aide à la décision. ELODIE s'appuie sur l'inventaire du cycle de vie et est conçu comme le premier module d'un outil plus complet d'évaluation environnementale des bâtiments conformes à la norme XP POI-020-3 (sur les indicateurs environnementaux et les méthodes de calcul associées pour l'évaluation environnementale des bâtiments). 
Une troisième échelle est aujourd'hui en cours de développement: l'échelle du quartier. Dans le cadre de notre étude, nous nous sommes appuyés sur l'outil à l'échelle quartier en cours de développement au sein du centre énergétique et de procédés de l'École des Mines Paristech.

Les relations entre le quartier et la ville, voire plus largement entre le quartier et le territoire, sont prises en compte par des données concernant les réseaux (rendement du réseau d'eau, réseau de chaleur éventuel, canalisations d'eau potable et d'eaux usées, etc.), les transports (présence de pistes cyclables, distance au transport en commun, etc.), les déchets (distances à une décharge, des usines de recyclage, un incinérateur, valorisation énergétique éventuelle, etc.), le climat, et, plus globalement, au niveau national, les moyens de production d'électricité et d'autres procédés.

Le bilan environnemental d'un quartier n'est pas simplement la somme des bilans des différents bâtiments qui le composent. D'une part, il faut tenir compte des interactions entre bâtiments, par exemple les masques solaires sur les différentes façades en fonction de la morphologie urbaine. D'autre part, certains équipements, comme une chaufferie alimentant un réseau de chaleur, peuvent être mutualisés. Enfin, les voiries et le système de transport peuvent être influencés par des paramètres comme la densité urbaine.

De même, le bilan d'un bâtiment n'est pas la somme des bilans de ses composants. Les interactions entre produits de construction ne peuvent pas être négligées. Par exemple, le rayonnement solaire transmis par une baie peut être stocké dans une dalle, ce qui réduit les besoins de chauffage et les impacts correspondants en fonction également des équipements. Le rayonnement peut être réfléchi par les murs et les cloisons, réduisant ainsi les consommations d'électricité pour l'éclairage artificiel, ce qui induit également une réduction des impacts environnementaux. En phase amont d'un projet, les produits ne sont pas précisément sélectionnés. L'ACV considère alors des données " génériques ", qui peuvent correspondre à des moyennes nationales ou européennes. Ces données sont précisées en fonction de l'avancement du projet: des données « spécifiques » peuvent être utilisées lorsqu'il s'agit par exemple de comparer plusieurs fabricants et/ou types de produit.

En résumé, cet outil, prolongement d'EQUER, permet d'inclure (Peuportier et al., 2006) :
- différents types de bâtiments (logements, magasins, bureaux, écoles, hôpitaux, hôtels, etc.),

- leurs infrastructures d'accès et des espaces publics (routes et rues, parcs de stationnement, espaces verts, etc.),

- des réseaux (système de distribution d'eau, égouts, gestion des déchets, chauffage urbain, etc.).

Le comportement des résidents - au travers des consommations d'eau et d'énergie, du traitement des déchets, du pourcentage de tri et de recyclage, etc. et les caractéristiques du site - au travers des distances de transport, du climat, des énergies utilisées pour la production d'électricité et de chauffage urbain, etc. - peuvent être pris en compte dans l'analyse.

Tout comme pour EQUER à l'échelle du bâtiment, cette analyse à l'échelle du quartier permet d'évaluer des indicateurs environnementaux pour les quatre phases du cycle de vie du quartier: construction (extraction des matières premières, production et transport des matériaux), utilisation (chauffage, éclairage public, consommation d'eau, etc.), rénovation (remplacement des composants : fenêtres, isolation, revêtements des bâtiments et des rues) et démolition (transport et traitement des déchets).

L'outil développé pour l'échelle quartier permet également différents usages (Peuportier et al., 2006) :

- Construction d'un nouveau quartier sur un terrain non bâti : Le modèle permet de montrer l'influence des choix de conception sur les impacts prévisibles pendant une durée d'analyse donnée (par exemple 80 ans). II inclut la fabrication, l'entretien et le remplacement des composants (par exemple, le choix de composants ayant une plus grande durabilité permet de réduire les impacts de « rénovation »), leur fin de vie (recyclage, mise en décharge, incinération, etc.), mais aussi la phase de fonctionnement du nouveau quartier (une forme urbaine plus compacte peut permettre de réduire les besoins de chauffage et les impacts correspondants). Des variantes de conception peuvent ainsi être comparées afin de guider les décisions.

- Démolition d'un îlot existant et construction de nouveaux bâtiments: Le calcul peut être effectué en deux phases: 
la modélisation de l'ancien îlot (les phases de gestion et de démolition sont alors prises en compte), puis celle du nouveau projet (cf. le cas précédent).

- Étude comparative entre la réhabilitation d'un îlot existant et sa « rénovation » (démolition puis reconstruction) : Le calcul de la démolition et de la reconstruction se fait selon le cas précédent. La réhabilitation nécessite également un double calcul. II est utile de faire un état des lieux des bâtiments existants, puis d'effectuer un deuxième calcul pour évaluer les projets après réhabilitation (l'étude de variantes peut également être intéressante). «Les impacts de la réhabilitation proprement dite peuvent être déduits par soustraction, les impacts ultérieurs (fonctionnement, " rénovation » et " démolition ») étant évalués par le deuxième calcul. »

\section{DESCRIPTION DES OUTILS D'ANALYSE}

L'analyse de cycle de vie à l'échelle du quartier s'appuie sur un ensemble de logiciels que sont ALCYONE, COMFIE-PLEIADES, EQUER et ARIADNE. Ces outils sont chainés les uns aux autres (Figure I).

ALCYONE est un outil de saisie graphique qui permet de décrire la géométrie d'un bâtiment (Salomon et al., 2005). Il permet de représenter un bâtiment ainsi que ses masques proches (bâtiments proches qui bloquent le rayonnement solaire direct). Les zones thermiques du bâtiment, zones dont le comportement thermique devrait être homogène, sont également définies dans ALCYONE.

COMFIE-PLEIADES est un logiciel de simulation thermique dynamique pour les bâtiments (Salomon et al., 2005). L'utilisateur peut importer une géométrie créée avec le logiciel de saisie graphique ALCYONE (il est possible d'importer des plans d'autres modeleurs graphiques). À partir de données sur les matériaux de construction, les scénarios d'occupation et la situation météorologique, le logiciel calcule les besoins en chauffage et en climatisation du bâtiment pour une période donnée (jusqu'à un an). Les résultats peuvent être désagrégés par zone thermique ou par période de temps. Le noyau de calcul COMFIE a été développé par le Centre Énergétique et Procédé des Mines de Paris (Peuportier et Blanc Sommereux, 1990), l'interface graphique PLEIADES par Izuba énergies.
EQUER est un outil d'évaluation de la qualité environnementale des bâtiments. Les besoins de chauffage (éventuellement de climatisation) calculés par le logiciel COMFIE sont exportés vers EQUER. Des données d'entrées supplémentaires doivent être fournies au logiciel pour effectuer l'analyse de cycle de vie, telles que le mix de production d'électricité, la possibilité ou non de recycler les déchets ménagers, le type de transport utilisé par les usagers, etc. Les résultats de l'ACV de chaque bâtiment sont présentés sous forme d'éco-profil, avec la possibilité de visualiser la contribution de chaque phase (constructionutilisation-rénovation-démolition) et de comparer jusqu'à quatre variantes d'un projet. Les éco-profils synthétisent les différents thèmes environnementaux abordés: l'épuisement des ressources (énergie primaire, eau, substances rares), l'effet de serre, la toxicité humaine, l'acidification, les déchets (radioactifs ou non), la qualité de l'air (smog et odeurs), la pollution de l'eau (eutrophisation et écotoxicité).

Le logiciel ARIADNE complète la chaine de logiciels bâtiment pour passer à l'échelle du quartier. Les résultats d'EQUER sont importés vers ARIADNE pour chaque type de bâtiment, en précisant leur nombre et les données supplémentaires concernant les espaces extérieurs et les réseaux qui doivent être fournies. Ainsi l'ACV quartier prend bien en considération les impacts des bâtiments, des infrastructures et des modes d'occupation des sols extérieurs. Les résultats sont aussi présentés sous la forme d'éco-profils.

Les données d'inventaire de fabrication des matériaux et d'autres procédés nécessaires à l'analyse de cycle de vie par les logiciels EQUER et ARIADNE sont issus de la base de données Ecoinvent' développée par des instituts de recherche suisses (Frischknecht et al., 2004).

Les impacts sur l'environnement d'un bâtiment ou d'un quartier sont évalués à travers différents indicateurs. Onze indicateurs sont évalués par EQUER pour chaque bâtiment du quartier, auxquels s'ajoutent quatre autres indicateurs pour passer à l'échelle quartier dans ARIADNE. Ces indicateurs peuvent être présentés en distinguant les différentes phases du cycle de vie du quartier présenté précédemment (construction, utilisation, rénovation et démolition).

Les onze indicateurs environnementaux évalués par EQUER à l'échelle du bâtiment sont :

\footnotetext{
' Dans cette étude, nous avons utilisé la version 1996 de la base de données Ecoinvent.
} 
I. L'effet de serre $\left(\mathrm{t} \mathrm{CO}_{2}\right.$ eq) : la contribution à l'effet de serre est mesurée par le potentiel de réchauffement global (Global Warming Potential GWP) de chacun des gaz à effet de serre.

2. L'eau utilisée $\left(\mathrm{m}^{3}\right)$ : représente la quantité d'eau douce puisée en $\mathrm{m}^{3}$.

3. L'énergie consommée (GJ): l'énergie consommée par le quartier est comptabilisée en énergie primaire sur l'ensemble du cycle de vie, de manière à prendre en compte l'énergie consommée depuis l'extraction des combustibles fossiles par exemple.

4. L'épuisement des ressources abiotiques (sans dimension): les ressources abiotiques étant les ressources naturelles non vivantes, c'est-à-dire les ressources minérales et fossiles, cet indicateur mesure la diminution estimée des stocks.

5. Les déchets inertes produits ( $t$ eq) : il s'agit des déchets issus des travaux de Voirie et Réseaux Divers - VRD (déblais de tranchée, pavés...), de construction, rénovation et démolition de bâtiments.

6. Les déchets radioactifs $\left(\mathrm{dm}^{3}\right)$ : cet indicateur représente le volume des différents déchets radioactifs produits (les déchets de catégorie $A$, de faible et moyenne activité, de catégorie $B$, de faible et moyenne activité mais contenant des éléments à durée de vie très longue, et de catégorie $\mathrm{C}$, de haute activité).

7. L'acidification ( $\mathrm{kg} \mathrm{SO}$ eq.) : l'acidification atmosphérique engendre des pluies acides très nocives pour la faune et la flore et est à l'origine du dépérissement des forêts. Ainsi cet indicateur mesure le potentiel d'acidification d'une substance.

8. L'eutrophisation ( $\mathrm{kg} \mathrm{PO} 4 \mathrm{eq})$ : l'apport excessif de substances nutritives (nitrates et phosphates entre autres) dans un milieu aquatique va accélérer le développement d'algues et de plantes aquatiques qui vont appauvrir le milieu en oxygène, et donc faire mourir les autres organismes vivants.

9. L'écotoxicité aquatique $\left(\mathrm{m}^{3}\right)$ : représente le volume d'eau polluée, par exemple par des métaux lourds ou des hydrocarbures. La pollution des eaux par les pesticides n'est pas prise en compte par cet indicateur car elle n'est pas reliée à la construction d'un quartier.

10. La formation d'ozone photochimique $\left(\begin{array}{lll}\mathrm{kg} & \mathrm{C}_{2} \mathrm{H}_{4} & \mathrm{eq}\end{array}\right)$ : l'ozone photochimique provient de la décomposition des composés organiques volatils (COV) sous l'action du soleil. Ce phénomène est à l'origine du «smog» d'été, particulièrement mauvais pour les voies respiratoires, d'où les politiques de circulation routière alternée lors des pics d'ozone en été.

II. La toxicité humaine ( $\mathrm{kg}$ hum.) : la toxicité humaine d'une substance est mesurée en prenant en compte les doses de substance reçues par les individus, c'est-à-dire le rapport entre la masse de polluants inhalée ou ingérée sur une durée donnée et la masse de l'individu.

Dans ARIADNE, le quartier est analysé à travers les onze indicateurs d'EQUER et quatre indicateurs supplémentaires :

I. Le potentiel de dégradation de la condition naturelle ( $\left.\mathrm{m}^{2} / a n n e ́ e s\right):$ impact en 'années' $x$ ' $\mathrm{m}^{2}$ d'occupation d'une surface naturelle équivalente'.

2. Le potentiel de dégradation de la condition naturelle due au changement d'utilisation ( $\mathrm{m}^{2} /$ années): évalue le changement d'utilisation du sol. Plus le sol utilisé dans le quartier est proche de sa condition naturelle, plus cet indicateur est bas.

3. La proportion de surface de terrain bâti dans le quartier (\%) : Mesure la densité du quartier, c'est-à-dire le rapport 'surface bâti' sur 'surface totale'.

4. L’imperméabilité moyenne du sol (\%) : la perméabilité aide à régénérer les nappes phréatiques et réduit la surcharge des réseaux de collecte des eaux pluviales.

La question de la fiabilité des outils d'ACV a été très vite posée. Trois outils ont été comparés dans le cadre du projet européen REGENER cité plus haut, puis une étude a été menée au sein de l'Agence Internationale de l'Énergie. L'inter-comparaison la plus complète à ce jour a été effectuée dans le cadre du réseau thématique européen PRESCO (Peuportier, 2004). Les résultats obtenus par huit outils ont été 
comparés tout d'abord dans un cas très simple, un cube en béton, ce qui a facilité l'analyse des écarts et la comparaison des données, des méthodes et des hypothèses de modélisation. Une deuxième étude de cas sur une maison réelle a ensuite permis de travailler sur un nombre plus élevé de paramètres.

Sur un indicateur comme la contribution au changement climatique, les écarts entre les outils se situent autour de plus ou moins $10 \%$, ce qui est du même ordre de grandeur que l'incertitude concernant les calculs de consommation énergétique. Sur d'autres aspects comme la santé, différents indicateurs sont utilisés (toxicité humaine, volumes critiques, années de vie perdues...), ce qui ne permet pas toujours de comparer les résultats. Des projets européens ultérieurs, ENSLIC Building et LORE LCA, en cours actuellement, donnent lieu à des échanges en vue d'identifier les bonnes pratiques et d'améliorer les modèles.

\section{LA ZAC CLAUDE BERNARD}

Nous avons testé ARIADNE sur un projet de ZAC parisienne. Ce test s'est fait a posteriori pour évaluer l'utilisation potentielle de l'ACV (faisabilité et informativité) à deux niveaux d'avancement d'un projet d'aménagement urbain. Les modélisations ont donc été effectuées en s'appuyant sur des données archivées ou collectées auprès de la maîtrise d'œuvre.

\section{I La ZAC Claude Bernard : élaboration et évolution}

Depuis 2002, le territoire Paris Nord Est (PNE) constitue avec dix autres sites le « Grand Projet de Renouvellement Urbain »parisien. Lancé par la Ville de Paris avec de nombreux partenaires, ce projet de grande envergure a pour objectif de repenser les relations entre la capitale et les communes limitrophes et surtout d'améliorer durablement la qualité de vie des habitants de la périphérie. Territoire de 200 hectares s'étendant entre les portes de la Chapelle et de la Villette, le secteur Paris Nord Est constitue actuellement le plus vaste secteur d'aménagement parisien.

Premier secteur opérationnel de PNE, la ZAC Claude Bernard (Figure 2), d'un peu moins de 15 hectares, est délimitée par le boulevard périphérique au nord, le boulevard Macdonald au sud, à l'est par le canal Saint Denis et à l'ouest par l'avenue de la Porte d'Aubervilliers. Les constructions se répartissent sur deux terrains : d'un côté la parcelle Claude Bernard et à l'Est l'îlot quai de la Charente. Nous nous sommes intéressés uniquement à la parcelle Claude Bernard.

Le programme d'aménagement est mixte et équilibré et prévoit la construction de logements, de bureaux et de locaux d'activités, d'un Établissement d'Hébergement pour Personnes Agées Dépendantes (EHPAD), d'une école polyvalente et d'une haltegarderie (Figure 2).

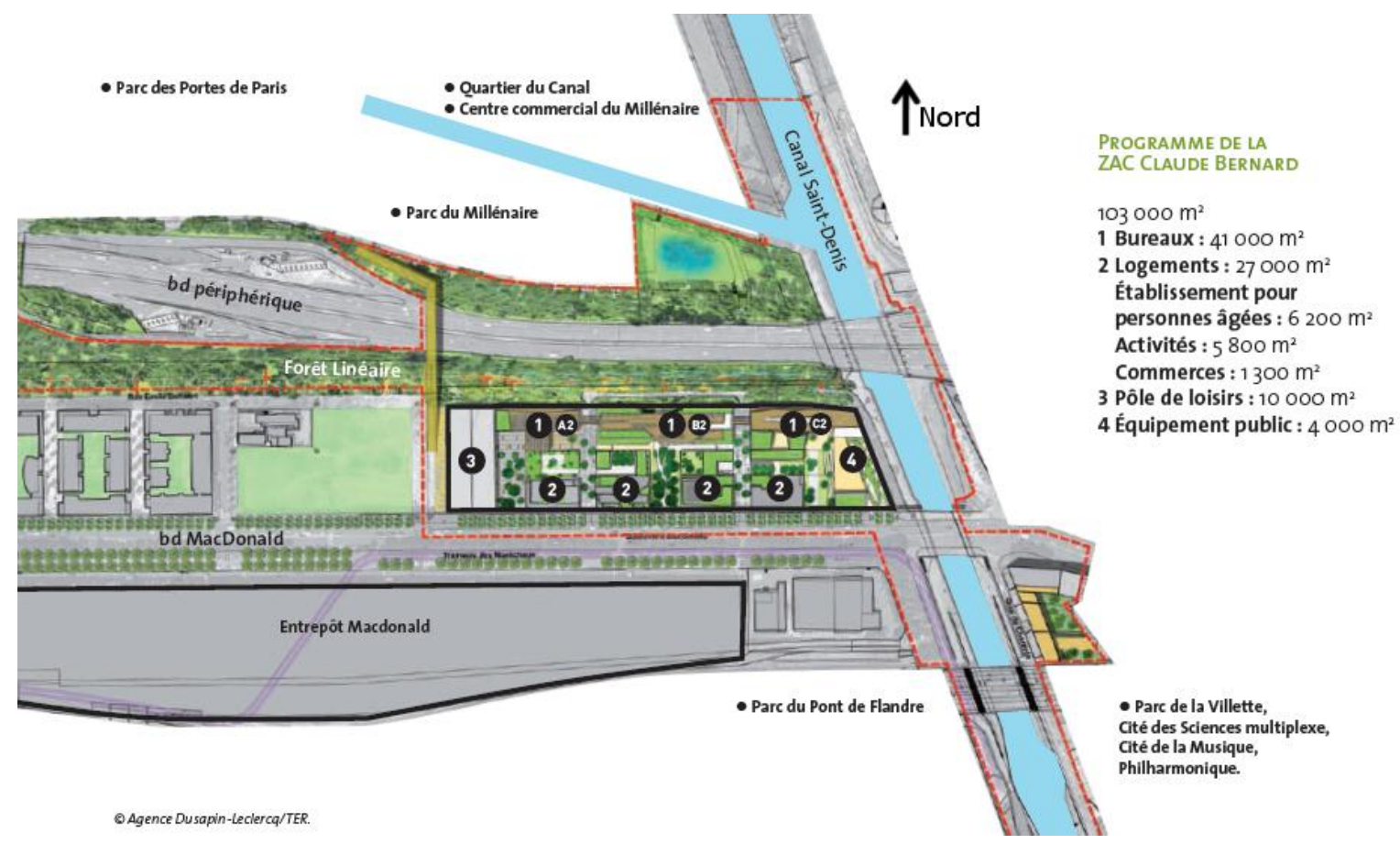

Source : Agence Dussapin\&Leclerq/TER

Fig. 2 - Programme de la ZAC Claude Bernard en 2009 
Ambitieux sur le plan environnemental, le projet met en œuvre un certain nombre d'innovations: le recours à la géothermie profonde (mise en œuvre par la CPCU, en partenariat avec la Région Ile-de-France et l'ADEME), la mutualisation des parcs de stationnement, la gestion des eaux pluviales de surface au moyen d'une noue, la création en bordure du canal d'une réserve écologique, ou encore l'évacuation des déblais de chantier par voie d'eau et l'acheminement des matériaux par voie ferrée.

L'élaboration du plan de masse s'est déroulée de manière continue et très peu de points de rupture ont été mis en évidence dans ce processus d'élaboration. Trois plans de masse distincts peuvent cependant être identifiés :

- Un premier plan de masse au stade de l'esquisse, pour lequel l'agence DussapinLeclercq a été choisie. Ce premier plan (Figure 3 ), qui date de 2003/2004, proposait deux mégastructures parallèles au périphérique sur lesquelles étaient disposées perpendiculairement plusieurs rangées de bâtiments. Cette conception traitait de manière innovante la question de la mixité fonctionnelle, puisque toutes les fonctions de la ZAC se retrouvaient dans une même structure.

- Un deuxième plan datant de 2007 (Figure 4) répartissait les bureaux dans des lots en forme de $\mathrm{T}$ de façon à ce que les bureaux, alignés le long du périphérique, et les immeubles de logements soient accessibles depuis le boulevard Macdonald. Les rez-dechaussée et les premiers étages des immeubles de logements étaient consacrés aux commerces et activités. Les bâtiments de bureaux forment une sorte de créneaux, de façon à créer une alternance d'immeubles et de cours ouvertes sur la forêt linéaire. Ce plan de masse a intégré un certain nombre de contraintes architecturales et environnementales. À cette occasion, les cahiers de prescriptions architecturales et paysagères, précisant entre autres le plan de masse et la volumétrie des bâtiments, ont été rédigés.

- Un troisième plan de masse (Figure 5), établi en 2010, conserve l'organisation des parcelles en macrolots $T$ pour les bureaux et $M$ pour les logements, mais contrairement au plan précédent, les bureaux deviennent des bâtiments très allongés, de manière à constituer un écran contre le bruit du périphérique. La programmation est restée identique au précédent.

\subsection{Variantes étudiées}

Afin d'analyser si, en phase initiale de conception, l'outil ARIADNE pourrait donner des éléments d'évaluation environnementale permettant d'aider la maitrise d'ouvrage dans ses décisions, nous avons réalisé quatre $A C V$ :

- Le plan de masse de 2007 avec les contraintes environnementales de 2007;

- Le plan de masse de 2010 avec les contraintes environnementales de 2007 ;

- Le plan de masse de 2010 avec la méthode constructive utilisée aujourd'hui;

- Le plan de masse de 2010 avec l'utilisation d'un pourcentage d'éco-matériaux.

Nous avons sélectionné, pour notre étude, les plans de masse de 2007 et 2010 , le premier ayant été rapidement abandonné. Pour simplifier les $A C V$, nous n'avons pas intégré dans notre étude le pôle de loisirs et la noue d'évacuation des eaux pluviales, ceux-ci n'ayant pas été modifiés entre les plans de masse de 2007 et de 2010.

\section{RÉSULTATS}

\section{I Comparaison des plans masse de 2007 et 2010}

Dans cette première expérimentation, nous allons comparer les impacts environnementaux du projet Claude Bernard selon le plan de masse choisi (2007 ou 2010 ) avec les contraintes environnementales de 2007, en phase initiale de conception. À cette phase, le programme des surfaces à construire a déjà été arrêté et un plan de masse établi. Les maîtres d'ouvrages, déjà désignés, n'ont pas encore présenté d'esquisses, et l'architecture des bâtiments n'a pas encore été définie. Seules des prescriptions architecturales et paysagères ont été formulées par l'architecte coordonnateur. 
EUE • Analyse de cycle de vie • c-9

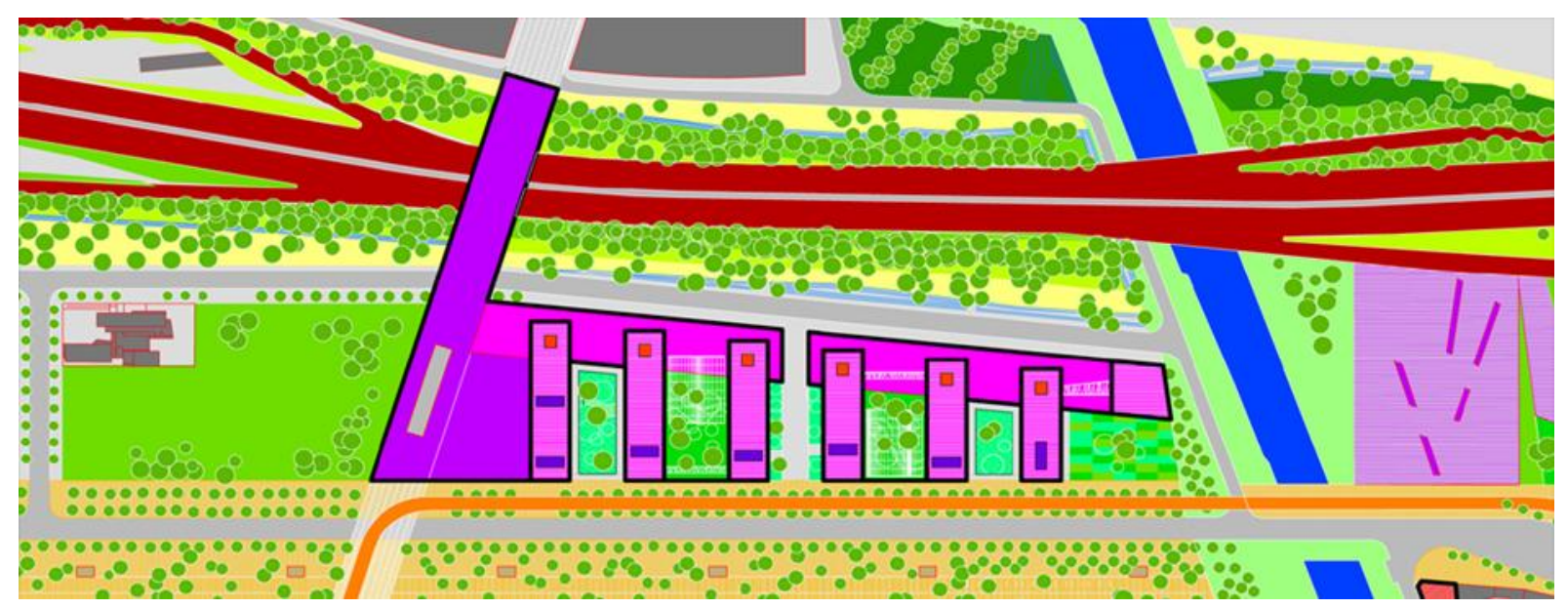

Source : Agence Dussapin\&Leclerq/TER

Fig. 3 - Plan de masse de 2003

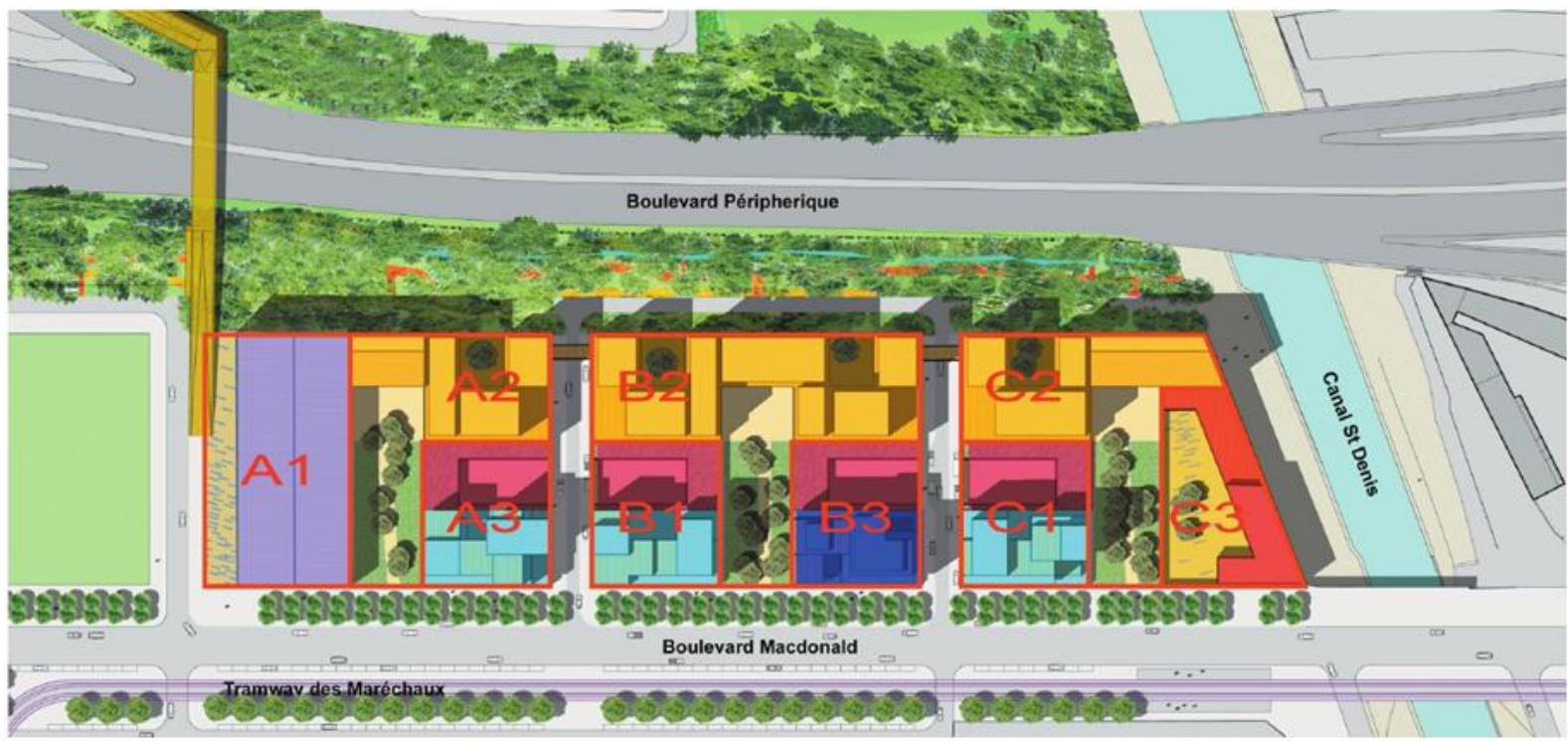

Les couleurs représentent la programmation (jaune= bureaux, bleu clair= logements, bleu foncé $=\mathrm{EHPAD}$, rose $=$ activités, violet $=$ commerces et pôle de loisir, rouge $=$ équipements)

Source : Agence Dussapin\&Leclerq/TER

Fig. 4 - Plan de masse de 2007 avec le découpage parcellaire 


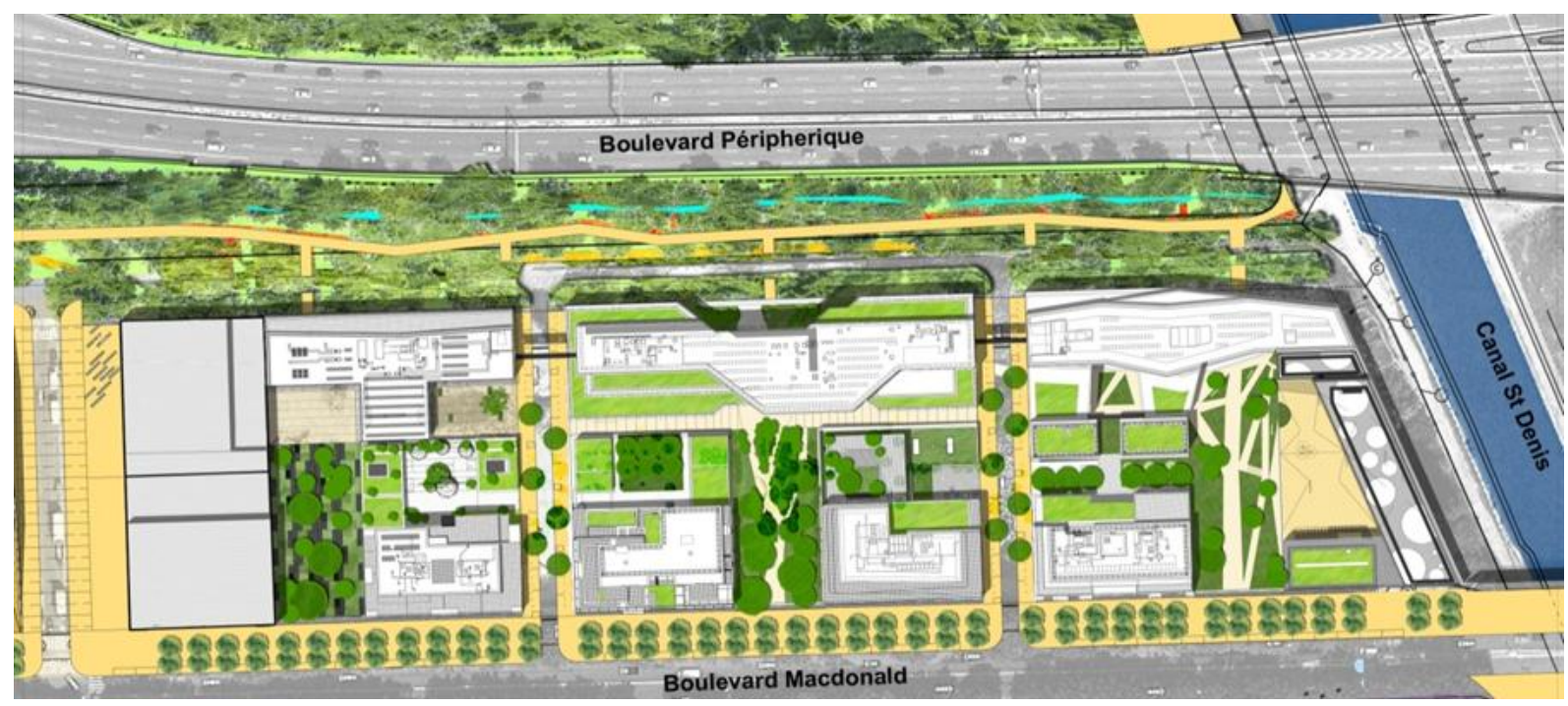

Source : Agence Dussapin\&Leclerq/TER

Fig. 5 - Plan de masse de 2010

L'objectif ici est de tester le potentiel d'aide à la décision de ARIADNE en phase initiale de conception, au moment du choix du plan masse.

Le calcul de l'ACV en cette phase amont de la conception a donc été réalisé sur la base des informations contenues dans les documents suivants :

- Le plan de masse de la ZAC Claude Bernard datant de 2006-2007;

- Les fiches de lots, comprenant des règles sur les hauteurs, la volumétrie, l'emprise au sol, les accès, etc.;

- L'étude d'aide à la décision énergétique réalisée par Cap Terre en 2007;

- La charte de développement durable de la SEMAVIP ${ }^{2}$ : elle précise les objectifs de

\footnotetext{
${ }^{2}$ Mission de la SEMAVIP (Société d'économie mixte de la Ville de Paris) dans le cadre du projet Paris Nord-Est: Dans le cadre d'une convention de prestation passée avec la SAS ParisNordEST, la SEMAVIP est chargée, notamment, des missions d'études techniques, environnementales et de programmation, des relations avec la Ville de Paris (interface avec le GPRU Paris-Nord-Est, adéquation du programme au PLU, passage du tramway, équipements publics, concertation avec les habitants), du contrôle de la mission de l'architecte coordonnateur et des travaux d'infrastructure et de VRD. La SEMAVIP assure en outre le montage juridique, la cession des droits à construire en application des décisions de ParisNordEST et, d'une manière générale, l'ensemble des études et la coordination indispensables à la bonne fin de l'opération.
}

développement durable et surtout de qualité environnementale. Ce document n'a pas de valeur obligatoire, mais il définit le niveau d'ambition environnementale retenu par les élus. Pour répondre à quatre grands enjeux de développement durable, la charte propose un certain nombre d'objectifs déclinés en actions. Ces quatre enjeux sont : I/ optimiser les ressources naturelles, 2/ réduire et maîtriser les nuisances, $3 /$ réguler la circulation et favoriser les modes de déplacements alternatifs, et $4 /$ assurer une dimension sociale et économique de la ZAC. Les objectifs développés dans la charte ont été traduits techniquement dans les cahiers de prescriptions environnementales, architecturales et paysagères. En 2007, ils exigeaient :

- L'obtention de la marque NF-HQE® - bâtiment tertiaire (2006) pour les bâtiments de bureaux: la priorité était donnée à la gestion de l'énergie et au confort acoustique (objectif : très performant), puis à la gestion de l'eau et à la qualité sanitaire de l'air (objectif : performant);

- L'obtention de la certification CERQUAL Habitat \& Environnement pour les logements : le profil A de la certification était exigé; 
- La mise en place pour les locaux d'activités, de commerces et de l'EHPAD d'une démarche reposant sur le principe d'opération pilote HQE尺 et bâtiments tertiaires mise en œuvre par Certivéa.

Le programme des surfaces à construire arrêté en 2005 est réparti comme indiqué dans le Tableau I.

$\mathrm{Au}$ regard des exigences environnementales formulées en 2007, les indicateurs d'impact environnemental à considérer en priorité sont : l'énergie consommée, l'effet de serre, la toxicité humaine, la production d'ozone photochimique, l'eutrophisation, l'eau utilisée, et l'écotoxicité aquatique.

À partir des fiches de lots datant de 2007, et notamment de la volumétrie, nous avons modélisé le plan de masse de 2007 dans le logiciel de géométrie ALCYONE (Figure 6). Le pôle de loisirs (lot $\mathrm{Al}$ ) a été uniquement modélisé sous forme de masque, car même s'il ne fait pas partie du périmètre de l'étude, son impact sur les apports solaires des bâtiments étudiés ne peut être négligé. Les surfaces de vitrage des différents bâtiments ont été modélisées en respectant les préconisations liées à la fonction des bâtiments. Les bâtiments ont été découpés en différente zones thermiques, c'est-à-dire des regroupements de pièces ayant un comportement thermique similaire et des scénarios de fonctionnement identiques, afin d'affiner les simulations de leurs besoins énergétiques.
Nous avons procédé de la même manière avec le plan de masse de 2010.

Dans cette expérimentation, les bâtiments des deux plans de masse ont été modélisés de manière à respecter les mêmes exigences en matière énergétique, celles de 2007 :

- conception bioclimatique des bâtiments;

- tendance vers l'auto-suffisance énergétique grâce au développement d'énergies renouvelables $(25 \%$ de leurs besoins énergétiques couverts par les énergies renouvelables);

- bonne isolation thermique des bâtiments selon la réglementation thermique de 2005 (20\% d'énergie consommée en moins par rapport à la valeur de référence (Cref)),

- installation d'appareils et d'équipements économes en énergie;

- chauffage non-électrique, énergétiquement performant et faiblement émetteur de Nox et de $\mathrm{CO}_{2}$.

Les bâtiments ont été modélisés de manière à respecter ces performances énergétiques avec des méthodes constructives actuelles « classiques » et des matériaux courants (béton et laine de roche comme isolant); les hypothèses retenues pour la composition des enveloppes sont précisées dans le tableau 2. Afin de simplifier la modélisation et en raison du manque

Tableau I

Affectation des surfaces en m $^{2}$ SHON en 2005

\begin{tabular}{llllllllr}
\hline & Bureaux & Logements & EHPAD & Activités & $\begin{array}{c}\text { Pôle de } \\
\text { loisirs }\end{array}$ & Commerces & Equipements & TOTAL \\
\hline Ilot A & 12000 & 6890 & 0 & 1700 & 10000 & 333 & 0 & 30923 \\
\hline Ilot B & 16000 & 6890 & 6000 & 2600 & 0 & 333 & 0 & 31823 \\
\hline Ilot C & 12000 & 6890 & 0 & 2200 & 0 & 334 & 5000 & 25424 \\
\hline TOTAL & 40000 & 20670 & 6000 & 6500 & 10000 & 1000 & 5000 & 93170 \\
\hline
\end{tabular}


d'information sur l'architecture des bâtiments en phase amont du projet, aucune paroi interne n'a été modélisée.

Le logiciel de simulation thermique dynamique PLEIADES-COMFIE simule les besoins en chauffage et climatisation d'un bâtiment pendant une année type. Pour réaliser cette simulation, il faut assigner au bâtiment des scénarios de vie (consigne de température, occupation, occultation, puissance dissipée, ventilation). Pour chaque bâtiment, un scénario de vie peut être choisi en fonction de sa destination (logements, bureaux, etc.). Ces hypothèses sont communes aux deux variantes testées ici.

Les consommations en énergie finale pour un an et les scénarios d'implantation des énergies renouvelables que nous avons utilisées sont décrites dans le tableau 3. Elles sont issues de l'étude d'aide à la décision énergétique réalisée par CapTerre (2006).

Les consommations de chauffage et d'eau chaude sanitaire (ECS) non prises en charge par des énergies renouvelables, le sont par le réseau de chauffage urbain de la Compagnie Parisienne de Chauffage Urbain (CPCU) dont le mix énergétique en 2007 est le suivant : $49 \%$ déchets, $27 \%$ gaz, $17 \%$ charbon, et $7 \%$ fioul $^{3}$. Selon les exigences en termes d'énergies renouvelables (25\% des besoins énergétiques couverts par les énergies renouvelables), les objectifs de consommation énergétique $(20 \%$ d'énergie consommée en moins par rapport à la valeur de référence) et les hypothèses retenues dans l'étude énergétique réalisée par CapTerre (Tableau 3), nous avons modélisé les bâtiments et simulé leurs besoins en énergie de chauffage et de climatisation; le tableau 4 récapitule l'ensemble des besoins énergétiques des bâtiments de la ZAC de manière à vérifier la cohérence de la modélisation avec les exigences du projet, avant de calculer les indicateurs d'impact environnemental.
Les consommations énergétiques des bâtiments (Tableau 4) sont en cohérence avec l'objectif RT 2005 moins $20 \%$. Les plots mixtes $\mathrm{Bl}$ et $\mathrm{Cl}$ (commerces, activités et logements) consomment moins que les bâtiments tertiaires ou l'EHPAD. Les bâtiments du plan de masse 2010 consomment en moyenne légèrement moins $\left(154, \mathrm{l} \mathrm{kWh} / \mathrm{m}^{2}\right.$.an contre 155,6 $\mathrm{kWh} / \mathrm{m}^{2}$.an). Les légères différences de forme et d'implantation des bâtiments doivent pouvoir expliquer cette faible différence de performance énergétique.

Dans cette étude, les déchets ménagers et le transport des occupants n'ont pas été pris en compte car ces aspects sont supposés indépendants du plan de masse. En d'autres termes, les variantes étudiées ici n'influencent pas le choix de moyen de transport des usagers ou la quantité des déchets qu'ils émettent. II serait intéressant de prendre en considération le transport des usagers pour comparer l'impact environnemental du choix entre deux sites d'implantation d'un même projet ou quand les variantes proposées pour un projet modifient l'accessibilité des bâtiments ou la place réservée à l'automobile.

Conformément aux résultats de la simulation des besoins énergétiques (Tableau 4), l'ACV comparative (Figure 7) montre que le plan de masse 2010 a un impact environnemental inférieur à celui de 2007. Dans cette comparaison, les matériaux de construction ainsi que le mix énergétique sont identiques pour les deux plans de masse. Les différences observées ne peuvent donc être dues qu'à la forme des bâtiments (compacité, quantité de matériaux de construction), leur implantation (orientation, phénomènes de masque solaire) et aux différences d'usage des sols entre les deux scénarios. La simulation montre ainsi qu'avec une durée de vie de 80 ans, nous obtenons une énergie consommée de 8236264 GJ (dont un peu moins de $98 \%$ pour la seule phase d'utilisation) pour le plan masse de 2010 et de

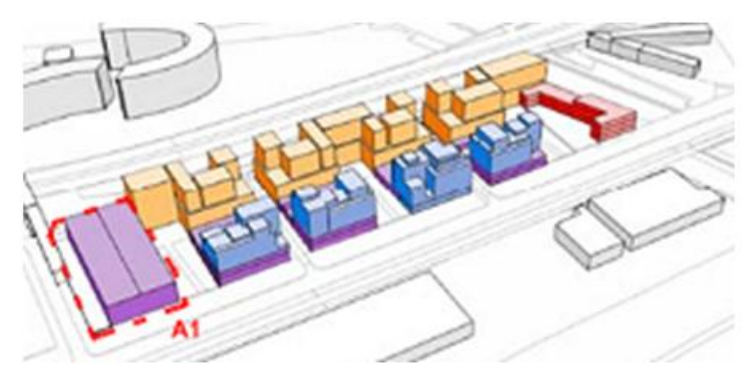

Volumétrie du projet en 2007 (Dussapin-Leclerq)

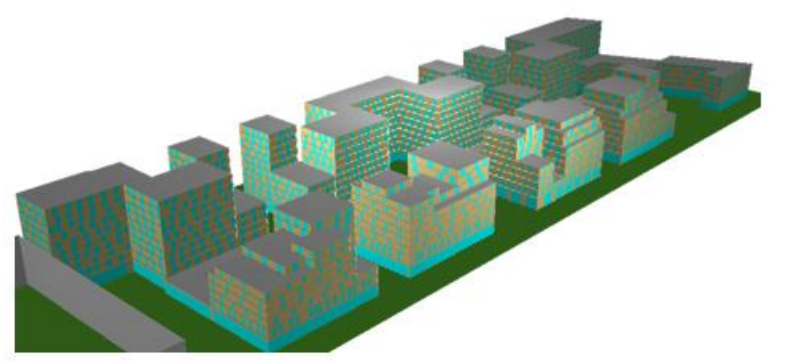

Vue 3D du plan de masse 2007 modélisé dans ALCYONE

Fig. 6 - De la volumétrie du projet à la modélisation dans ALCYONE 
$8269613 \mathrm{GJ}$ (dont également un peu moins de $98 \%$ pour la seule phase d'utilisation) pour celui de 2007. Pour l'effet de serre, les valeurs sont respectivement, pour les plans masses de 2010 et de 2007, de 163601 $\mathrm{tCO}_{2}$ (dont $92 \%$ pour la seule phase d'utilisation) et de $184119 \mathrm{tCO}_{2}$ (dont 93\% pour la seule phase d'utilisation).

Nous avons considéré que l'utilisation initiale du sol était « continuous urban fabric », c'est-à-dire une occupation équivalente à un centre-ville. Cette hypothèse est commune aux deux simulations, puisque nous étudions deux variantes d'un même projet sur un même site. L'utilisation du sol ne varie pas beaucoup entre les variantes (Tableau 5) avec respectivement, pour le plan masse de 2007 et celui de 2010 , 51\% et $56 \%$ de part de terrain bâti et une imperméabilité moyenne du sol de $86 \%$ et $88 \%$. Les résultats des indicateurs d'ARIADNE sont donc très proches d'un plan de masse à l'autre. Le potentiel de dégradation de la condition naturelle lié au changement d'utilisation du sol est amélioré par les deux variantes. Le logiciel ARIADNE associe à chaque utilisation du sol une valeur type de potentiel de dégradation de la condition naturelle, allant de $0, I$ pour des espaces naturels à I pour des bâtiments. Ainsi le projet dans ses deux variantes offre une surface de sol dont l'utilisation est plus proche d'un espace naturel que l'utilisation initiale du site.

Le plan de masse 2010 comporte $1000 \mathrm{~m}^{2}$ de surface d'allée ou parking en plus, mais $2000 \mathrm{~m}^{2}$ d'espaces vert en moins, ce qui explique que son potentiel de dégradation de la condition naturelle soit supérieur à celui de 2007 (45825 m².années contre $39500 \mathrm{~m}^{2}$.années).

\subsection{Comparaison de variantes sur le plan de masse de 2010}

Dans cette seconde expérimentation de l'ACV sur la ZAC Claude Bernard, nous considérons uniquement le macro lot $M$ constitué des plots mixtes ( $A 3, B I, B 3$ et $\mathrm{CI}$ ) longeant le boulevard Macdonald. Cette partie de l'étude a été réalisée alors que la ZAC Claude Bernard était en travaux, ce qui a rendu particulièrement difficile la collecte d'informations auprès des maîtres d'œuvre. Nous nous sommes donc limités, dans cette seconde expérimentation, à l'analyse des plots mixtes et de leur environnement proche.

Nous analysons cette fois le plan de masse arrêté définitivement en 2010. Cette ACV se situe donc en « phase finale de la conception », c'est-à-dire que la programmation est arrêtée, que le plan de masse ne sera plus modifié, que l'architecture des bâtiments a été décidée et que le choix des matériaux est finalisé. L'objectif ici est d'observer le potentiel d'aide à la décision de l'outil ARIADNE en phase finale de conception.

Nous allons donc comparer, pour le plan de masse de 2010, les solutions techniques (équipements et matériaux) proposées par la maîtrise d'œuvre pour les plots mixtes à une référence haute en matière environnementale que nous appelons «meilleures pratiques $»$.

Affichés dans le Plan Climat de la Ville de Paris (2007), les objectifs de performance énergétique ont été légèrement renforcés par rapport à 2006 : réduction d'au moins $20 \%$ en moyenne des consommations énergétiques en référence à la réglementation thermique RT 2005. Recours aux énergies renouvelables: le pourcentage de consommation d'énergies renouvelables sera d'au moins $25 \%$ pour toutes les constructions.

L'objectif en matière d'énergies renouvelables a été dépassé, et atteint un taux de 30\% d'ENR grâce au recours à la géothermie profonde par la CPCU. L'énergie qui devrait approvisionner le réseau de chaleur de la CPCU Paris Nord Est sera donc issue du mix suivant: $32 \%$ géothermie, $30 \%$ valorisation thermique des ordures ménagères, $18 \%$ gaz naturel, $13 \%$ charbon, $5 \%$ fuel et $2 \%$ électricité ${ }^{4}$.

Dans le dossier de candidature à l'appel à projet « Nouveaux Quartiers Urbains » de 2009 lancé par le Conseil Régional d'lle-de-France, les objectifs de performances énergétiques préconisés pour la ZAC Claude Bernard s'élevaient à :

- Cep< 65kWh/m².an pour l'habitat (Bâtiment Basse Consommation - BBC);

- RT 2005 - 25\% pour les bâtiments tertiaires;

- RT 2005 - 50\% pour les équipements publics (BBC).

Ces derniers objectifs ont été atteints pour les plots mixtes (commerces, activités, logements le long du boulevard Macdonald).

\footnotetext{
${ }^{4}$ Chiffres issus de la demande par la CPCU d'agrément de la méthode de justification d'utilisation du système de réseau de chaleur pour Paris Nord-Est datant du I er octobre 2008.
} 
EUE • Analyse de cycle de vie $\bullet$ c- 14

Tableau 2

Hypothèses sur les matériaux de construction en phase initiale de conception

INTITULE

\section{COMPOSITION}

CARACTERISTIQUES

THERMIQUES

\begin{tabular}{lll}
\hline & Enduit plâtre $3 \mathrm{~cm}$, laine de roche 10 & $\mathrm{R}$ paroi=2,91 \\
& $\mathrm{cm}$, béton lourd $16 \mathrm{~cm}$, carreau de & \\
& plâtre $1 \mathrm{~cm}$ & \\
\hline PLANCHERS & Laine de roche $10 \mathrm{~cm}$, béton lourd 16 & $\mathrm{R}$ paroi=2,53 \\
& $\mathrm{cm}$ & \\
\hline PLANCHERS INTERMEDIAIRES & Béton $16 \mathrm{~cm}$, laine de roche $3 \mathrm{~cm}$ & $\mathrm{R}$ paroi $=0,82$ \\
\hline TOITURE & Calcaire dur $5 \mathrm{~cm}$, feutre bitumeux 2 & $\mathrm{R}$ paroi $=2,59$ \\
& $\mathrm{~cm}$, laine de roche $10 \mathrm{~cm}$, béton lourd & \\
\hline FENETRES & $16 \mathrm{~cm}$ & $\mathrm{Uw}=2,76 \mathrm{~W} /\left(\mathrm{m}^{2} . \mathrm{K}\right)$ \\
\hline
\end{tabular}

Tableau 3

Hypothèses sur les consommations annuelles en énergie finale et sur l'implantation des énergies renouvelables

\begin{tabular}{|c|c|c|c|c|}
\hline & Logements & $\begin{array}{l}\text { Locaux } \\
\text { tertiaires }\end{array}$ & EHPAD & Ecole \\
\hline Consommation pour l'ECS (kWh/m²) & 32,5 & 9 & 58 & 19,5 \\
\hline $\begin{array}{l}\text { Consommation pour l'éclairage et } \\
\text { autres postes }\left(\mathrm{kWh} / \mathrm{m}^{2}\right)\end{array}$ & 20 & 40 & 60 & 40 \\
\hline Couverture pour le solaire thermique & $30 \%$ & $21 \%$ & $45 \%$ & $21 \%$ \\
\hline Couverture pour le photovoltaïque & $10 \%$ & $34 \%$ & $15 \%$ & $34 \%$ \\
\hline
\end{tabular}


EUE • Analyse de cycle de vie • c-I5

Tableau 4

Consommations énergétiques annuelles $\left(\mathrm{kWh} / \mathrm{m}^{2}\right)$ simulées de plusieurs bâtiments ${ }^{5}$ de la ZAC Claude Bernard selon les plans de masse de 2007 et de 2010

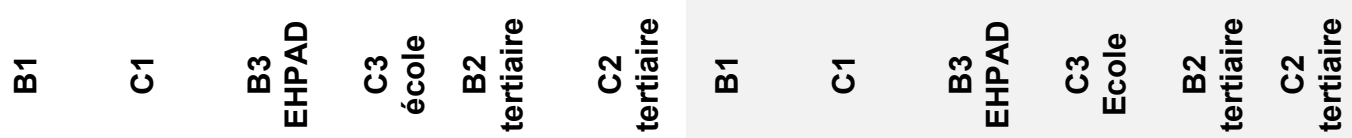

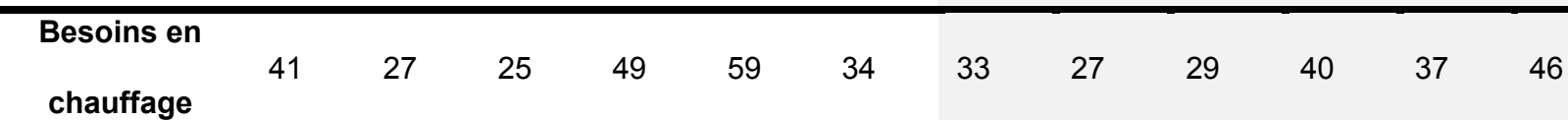

\begin{tabular}{|c|c|c|c|c|c|c|c|c|c|c|c|c|}
\hline Consommation & & & & & & & & & & & & \\
\hline $\begin{array}{l}\text { primaire } \\
\text { chauffage }\end{array}$ & 34 & 22 & 21 & 41 & 48 & 28 & 27,5 & 22,5 & 24 & 33 & 31 & 38 \\
\hline $\begin{array}{l}\text { Besoins en } \\
\text { climatisation }\end{array}$ & 3 & 4 & 8 & 2 & 9 & 1 & 3 & 3 & 4 & 6 & 3 & 3 \\
\hline Consommation & & & & & & & & & & & & \\
\hline $\begin{array}{l}\text { primaire clim } \\
\qquad(\mathrm{cop}=2)\end{array}$ & 3 & 5 & 10 & 2,58 & 12 & 1,3 & 4 & 4 & 5 & 8 & 4 & 4 \\
\hline $\begin{array}{c}\text { Consommation } \\
\text { finale ECS }\end{array}$ & 26 & 25 & 58 & 19,5 & 9 & 9 & 26 & 25,5 & 58 & 19,5 & 9 & 9 \\
\hline $\begin{array}{c}\text { Consommation } \\
\text { primaire ECS }\end{array}$ & 21 & 21 & 48 & 16 & 7,5 & 7,5 & 22 & 21 & 48 & 16 & 7,5 & 7,5 \\
\hline $\begin{array}{l}\text { Consommation } \\
\text { finale autres }\end{array}$ & 26 & 26 & 60 & 40 & 40 & 40 & 25,5 & 26 & 60 & 40 & 40 & 40 \\
\hline $\begin{array}{l}\text { Consommation } \\
\text { primaire autres }\end{array}$ & 66,6 & 67 & 155 & 103 & 103 & 103 & 65,5 & 67 & 155 & 103 & 103 & 103 \\
\hline TOTAL & 125 & 115,5 & 234 & 163 & 156 & 140 & 119 & 115 & 232 & 160,5 & 145 & 153 \\
\hline
\end{tabular}

${ }^{5}$ En raison de contraintes de calendrier et de problèmes techniques, la vérification de la cohérence des consommations énergétiques avec les objectifs de 2007 n'a pas pu être réalisée pour l'ensemble des bâtiments de la ZAC. En revanche, l'ACV a été effectuée avec tous les bâtiments. 
EUE • Analyse de cycle de vie • c-16

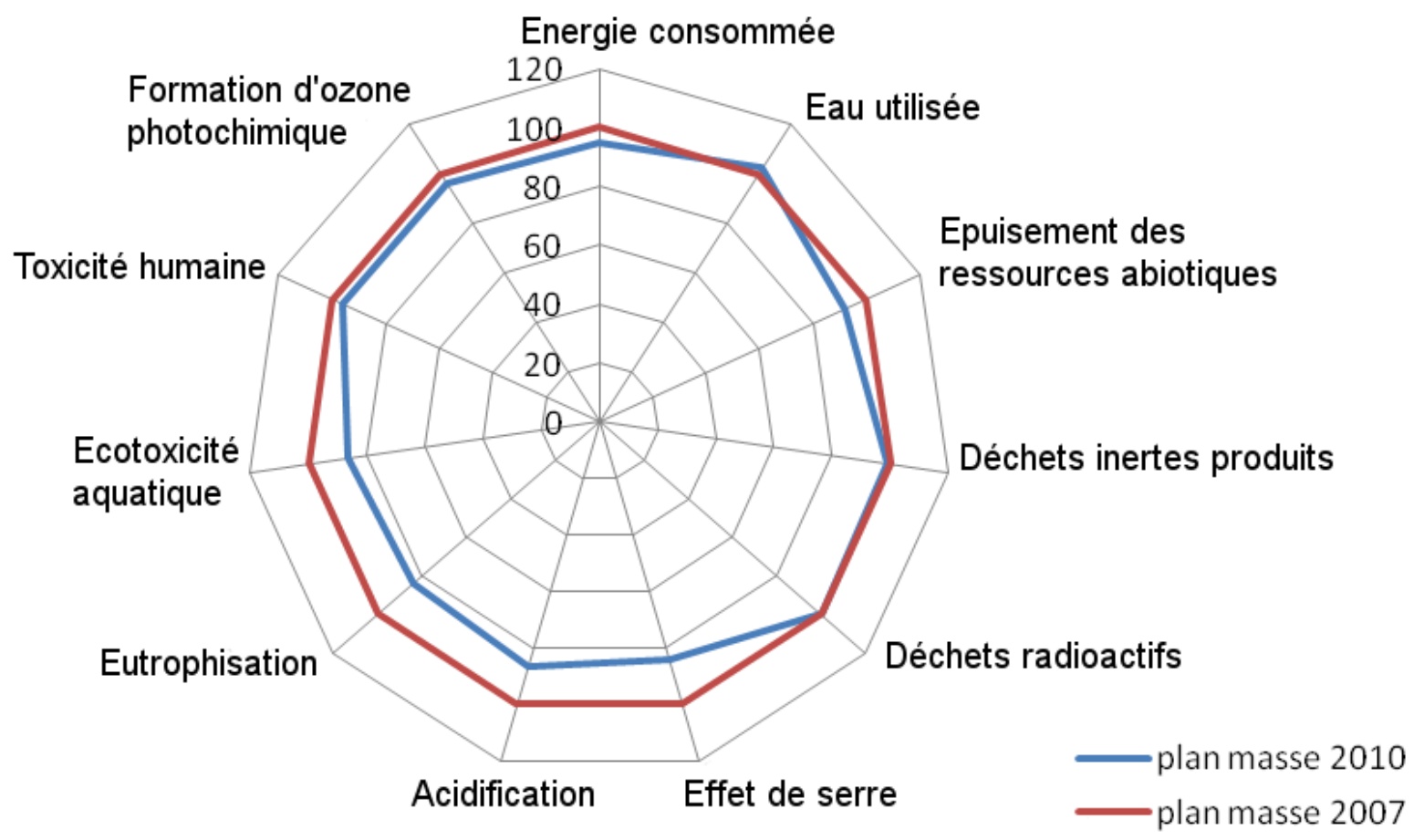

Fig. 7 - Analyse de cycle de vie comparative des plans de masse de 2007 et 2010 (logiciel ARIADNE).

Tableau 5

Résultats de la comparaison spécifique à l'échelle quartier entre les plans de masse de 2007 et 2010 (logiciel ARIADNE)

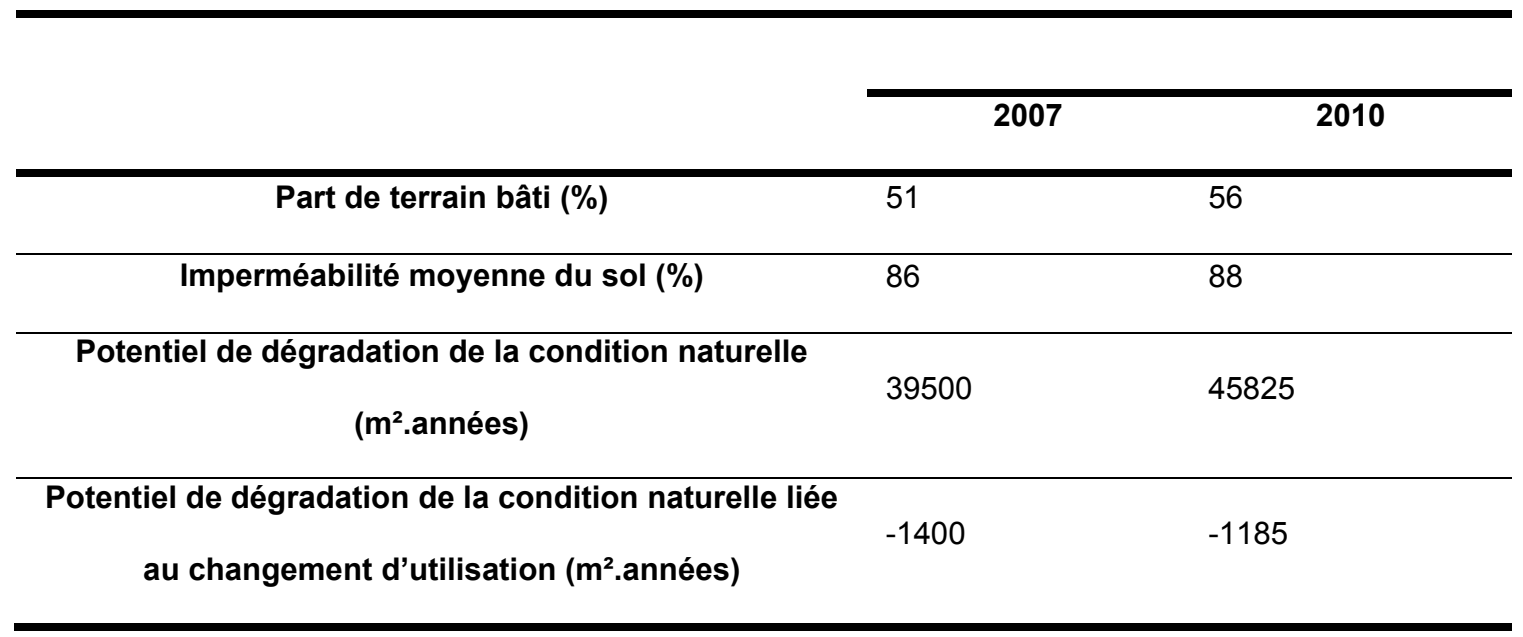


EUE • Analyse de cycle de vie • c-I 7

Tableau 6

Matériaux de construction des plots mixtes AI, B I, B3 tels qu'ils sont construits en 2010

\begin{tabular}{|c|c|c|}
\hline INTITULE & COMPOSITION & $\begin{array}{c}\text { CARACTERISTIQUES } \\
\text { THERMIQUES }\end{array}$ \\
\hline FACADES & $\begin{array}{l}\text { Plâtre } 1 \mathrm{~cm} \text {, polystyrène } 10 \mathrm{~cm} \text {, béton } \\
\qquad 20 \mathrm{~cm}\end{array}$ & R paroi $=2,70$ \\
\hline PLANCHER BAS & $\begin{array}{c}\text { Béton } 23 \mathrm{~cm}, \\
\text { Polystyrène } 15 \mathrm{~cm} \\
\end{array}$ & R paroi $=3,98$ \\
\hline PLANCHERS INTERMEDIAIRES & $\begin{array}{c}\text { Béton } 5 \mathrm{~cm} \text {, béton } 23 \mathrm{~cm}, \\
\text { polystyrène } 9,5 \mathrm{~cm}\end{array}$ & $\mathrm{R}$ paroi= 2,60 \\
\hline TOITURE & $\begin{array}{l}\text { feutre bitumeux } 1 \mathrm{~cm} \text {, polyuréthane } \\
\qquad 10 \mathrm{~cm} \text {, béton } 20 \mathrm{~cm}\end{array}$ & $\mathrm{R}$ paroi $=3,46$ \\
\hline FENETRES & $\begin{array}{c}\text { Double vitrage avec lame d'argon } \\
\text { cadre bois }\end{array}$ & $\mathrm{Uw}=1,42 \mathrm{~W} /\left(\mathrm{m}^{2} \cdot \mathrm{K}\right)$ \\
\hline
\end{tabular}

\section{Tableau 7}

Solutions techniques proposées en $\mathbf{2 0 0 8}$ pour améliorer les performances environnementales des plots mixtes

\begin{tabular}{|c|c|}
\hline Énergie & $\begin{array}{l}\text { - Connexion au réseau de chaleur de la CPCU } \\
\text { - Isolation par l'extérieur des bâtiments et rupteurs de ponts thermiques } \\
\text { - } \quad \text { Volets roulants extérieurs sur toutes les baies vitrées } \\
\text { - } \quad \text { Optimisation de l'éclairage naturel par puits de lumière pour les séjours et les } \\
\text { cuisines } \\
\text { - } \quad \text { Compteurs individuels } \\
\text { - Détecteurs de présence reliés à des minuteries dans les locaux techniques }\end{array}$ \\
\hline $\begin{array}{l}\text { Ventilation \& confort } \\
\text { d'été }\end{array}$ & $\begin{array}{l}\text { - Volets roulants extérieurs sur toutes les baies vitrées } \\
\text { - Pas de climatisation } \\
\text { - Ventilation simple flux }\end{array}$ \\
\hline Bruit & - Protection acoustique des façades exposées au Boulevard Périphérique \\
\hline Gestion de l'eau & $\begin{array}{l}\text { - Mise en place d'équipements hydro-économes ( } 30 \% \text { d'eau économisée) } \\
\text { - Végétalisation des toitures-terrasses des RDC } \\
\text { - } \quad \text { Récupération de l'eau de pluie des toitures pour arrosage des espaces verts et } \\
\text { nettoyage des locaux communs } \\
\text { - } 20 \% \text { de la surface de chaque lot conservé en pleine terre }\end{array}$ \\
\hline Qualité de l'air & $\begin{array}{l}\text { - Revêtements intérieurs éco-labellisés et choisis en fonction de leur taux d'émission } \\
\text { de Composés Organiques Volatils }\end{array}$ \\
\hline Chantier & - Mise en place d'une « Charte de Chantier Propre » \\
\hline
\end{tabular}


EUE • Analyse de cycle de vie • c- 18

Tableau 8

Comparaison des consommations énergétiques pour les deux variantes du plan de masse 2010

\begin{tabular}{|ccccc|}
\hline \multirow{2}{*}{ PM 2010} & B1 & B3 & A3 \\
& Besoins de chauffage $\left(\mathbf{k W h} / \mathbf{m}^{2}\right)$ & 17 & 31 & 23 \\
\cline { 2 - 4 } Meilleures pratiques & Besoins clim $\left(\mathbf{k W h} / \mathbf{m}^{\mathbf{2}}\right)$ & 11 & 9 \\
\cline { 2 - 4 } & Besoins de chauffage $\left(\mathbf{k W h} / \mathbf{m}^{\mathbf{2}}\right)$ & 5 & 7 \\
\cline { 2 - 4 } & Besoins de clim $\left(\mathbf{k W h} / \mathbf{m}^{\mathbf{2}}\right)$ & 11 & 9 \\
\hline
\end{tabular}

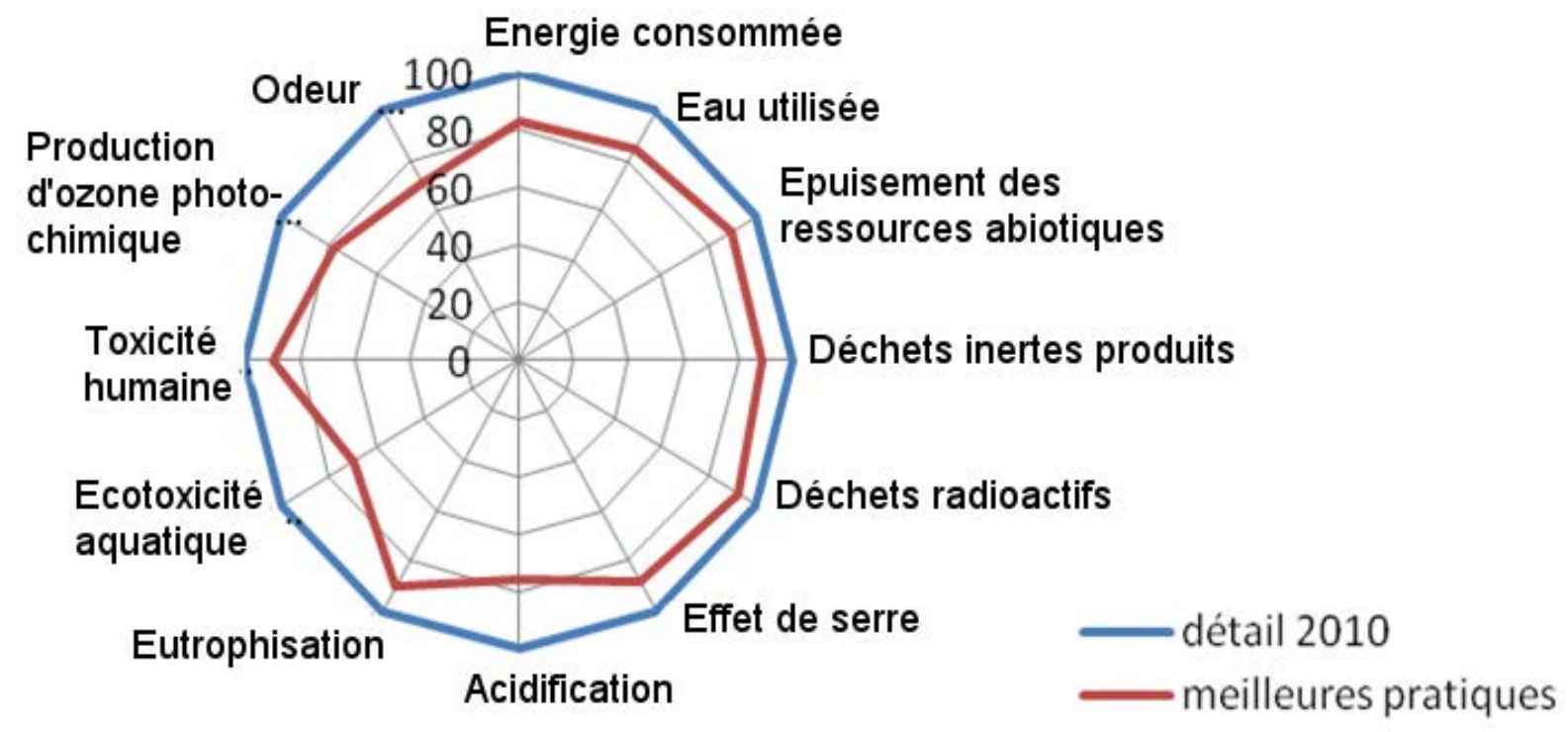

Fig. 8 - Analyse de cycle de vie comparative des deux variantes pour le plan de masse 20 IO (logiciel ARIADNE) 
Pour calculer l'ACV en phase finale, nous avons modélisé les bâtiments tels qu'ils devraient être construits, puisque que nous avons déterminé les hypothèses à partir des informations disponibles à l'heure actuelle (juin 2010). Pour collecter les données nécessaires à la réalisation de l'ACV, nous avons récupéré directement les plans, coupes, façades et informations sur les matériaux et équipements auprès des maitres d'œuvre. Les matériaux de construction des plots mixtes $(\mathrm{A} 3, \mathrm{BI}, \mathrm{B} 3)$ et leurs caractéristiques thermiques sont récapitulés dans le tableau 6.

Un certain nombre de solutions techniques (tableau 7) ont été proposées pour les plots mixtes (commerces, activités, logements le long du boulevard Macdonald) et sont disponibles dans les notices environnementales des permis de construire.

Les solutions techniques ont été intégrées au mieux lors des modélisations dans les différents logiciels de l'ACV.

Nous avons comparé les plots mixtes du plan de masse 2010 de la ZAC Claude Bernard, en faisant varier les caractéristiques de construction des bâtiments.

La variante «PM 2010 » correspond aux bâtiments du plan de masse 2010 tels qu'ils sont en train d'être construits. Dans la variante « meilleures pratiques », l'isolant laine de roche a été remplacé par de la laine de chanvre ${ }^{6}$, en conservant la même performance thermique, et la présence d'une ventilation double flux a été simulée.

Nous observons logiquement une baisse de l'impact environnemental au profit de la variante « meilleures pratiques » (figure 8). Le gain du point de vue de l'écotoxicité peut en partie s'expliquer par le remplacement du polystyrène par de la laine de chanvre. La différence de performance pour les indicateurs énergie et effet de serre est principalement liée à la présence de la ventilation double flux dans la variante « meilleures pratiques », comme le montrent les écarts en termes de besoins de chauffage (tableau 7).

La simulation montre ainsi qu'avec une durée de vie de 80 ans, nous obtenons une énergie consommée de $2535680 \mathrm{GJ}$ pour le plan masse de 2010 et de

\footnotetext{
${ }^{6}$ La laine de chanvre est considérée comme un éco-matériau. Le chanvre est une plante et de ce fait une ressource naturelle et renouvelable, qui d'après Oliva (2006) est « un des meilleurs compromis techniques, économiques et écologiques pour une isolation de qualité $»$.
}

2100000 GJ pour « meilleures pratiques». Pour l'effet de serre, les valeurs sont, respectivement pour les plans masses de 2010 et «meilleures pratiques », de $35386 \mathrm{tCO}_{2}$ et de $31168 \mathrm{tCO}_{2}$.

Les performances environnementales de la variante «meilleures pratiques » auraient pu être encore améliorées en augmentant la part d'énergies renouvelables.

\section{CONCLUSION}

Une des particularités des projets d'aménagement est qu'ils s'étalent dans le temps alors que les études techniques sont souvent ponctuelles. Si les projets d'aménagement se font dans la durée, les décisions influant sur la qualité environnementale du projet sont elles aussi diluées dans le temps. Dans ce contexte, l'analyse de cycle de vie peut venir accompagner le projet dans la durée et pour chaque étape liée à une prise de décision. Par leur fonctionnement, les logiciels utilisés pour l'étude semblent adaptés à cette contrainte. En effet, une fois le fichier de simulation créé, celui-ci peut évoluer en fonction d'informations nouvelles, de contraintes supplémentaires ou de décisions à prendre (comparaison de variantes, etc.).

Le recueil des données nécessaires à la simulation est une étape néanmoins essentielle et chronophage de l'analyse de cycle de vie. Dans le cas d'un projet d'aménagement, l'importante quantité d'informations nécessaires ainsi que la diversité des acteurs (maitre d'ouvrage, maitre d'œuvre, assistant à la maitrise d'ouvrage, etc.) rend difficile la collecte de données. Ce frein permet de souligner l'importance de la pédagogie autour de la démarche afin d'encourager tous les acteurs concernés à contribuer et à participer à la démarche, le risque étant sinon de fonctionner avec des données dégradées.

Les simulations, effectuées à différentes étapes de l'avancement d'un projet, permettent de mettre en avant les apports et limites de l'ACV quant à l'évaluation environnementale et sa capacité à aider les maitres d'ouvrage dans leur prise de décision. En phase amont d'un projet, l'ACV peut permettre de comparer différentes morphologies urbaines (plans de masse) sur des critères plus globaux que la simple analyse énergétique. D'autre part, des alternatives énergétiques (géothermie dans le cas étudié ici) peuvent être évaluées sur la base de leur contribution à la réduction des impacts environnementaux. L'ACV peut s'avérer également utile à une étape plus avancée de la prise de décision, quand il s'agit de comparer différents matériaux ou équipements. 
L'approche par l'analyse de cycle de vie permet de compléter les résultats des études techniques classiquement effectuées pour les projets d'aménagement (étude d'impact en environnement, bilan carbone, etc.). Les opportunités offertes par cette méthode sont à la fois techniques et pédagogiques. L'ACV permet d'élargir le débat au-delà des questions énergétiques et au-delà de la seule phase d'utilisation du bâtiment. Elle propose de plus une étude intégrée du bâtiment au quartier. L'ACV permet également d'obtenir des informations chiffrées facilitant la communication et la sensibilisation.

L'ACV reste cependant un outil très technique. La méthode de l'analyse de cycle de vie et les logiciels utilisés relèvent du domaine de l'ingénierie. Ainsi, tant sur la pratique des logiciels que sur l'interprétation des résultats, des connaissances scientifiques sont nécessaires. Le nombre important d'indicateurs, s'il permet au logiciel d'être très complet, rend difficile l'interprétation des résultats. Pour le maître d'ouvrage qui doit prendre une décision, cela implique d'effectuer une hiérarchisation des indicateurs, qui doit s'appuyer sur une bonne connaissance de ces derniers ainsi que des sciences et techniques du bâtiment. Cette complexité technique du logiciel peut-être un éventuel verrou à l'appropriation de l'outil ACV par les différents acteurs de l'aménagement, et notamment la maîtrise d'ouvrage.

De plus, la signification des indicateurs d'ARIADNE, et donc leur interprétation, ne sont pas évidentes. Ils mériteraient d'être enrichis d'indicateurs de biodiversité par exemple, afin de mieux évaluer l'impact sur la faune, la flore et la santé humaine de la disparition ou de la création d'un $\mathrm{m}^{2}$ d'espace vert.

La chaîne de logiciels d'analyse de cycle de vie utilisée dans le cadre de l'étude peut donc se révéler un outil pertinent dans la conduite d'un projet urbain. Les logiciels peuvent fournir des informations quantitatives utiles aux différentes étapes clés, et donner ainsi des éléments d'aide à la décision à la maîtrise d'ouvrage tout au long du projet. Trois points nous semblent toutefois importants à souligner :

- la difficulté d'interprétation des résultats, du fait de la grande variété des indicateurs: cette complexité de l'information recueillie à l'aide des logiciels peut aller à l'encontre de l'objectif initial, à savoir apporter des éléments d'aide à la décision.

- l'absence de certaines données pourtant importantes dans le menu des logiciels: certaines technologies, par exemple le solaire thermique et photovoltaique sont encore absents. Une intégration plus rapide des nouvelles technologies, des nouveaux matériaux, etc. aux logiciels serait donc souhaitable.

- le manque de clarté dans la signification de certains indicateurs spécifiques au logiciel ARIADNE (échelle quartiers): le lien entre ces indicateurs et les autres n'est $a$ priori pas évident.

\section{PERSPECTIVES}

Cette étude constitue la première expérience d'analyse de cycle de vie d'un projet d'aménagement pour la Ville de Paris. Les différentes études de cas fictifs ou concrets présentés dans cet article et dans Tardieu et al. (20II) ont montré que la méthode offre des opportunités nouvelles, qui font aujourd'hui défaut aux collectivités pour la prise en considération des impacts environnementaux d'un projet d'aménagement. L'intégration d'un suivi par l'analyse de cycle de vie dans le cahier des charges des projets d'aménagement est envisageable. Elle pourrait être à la charge de l'équipe de maîtrise d'œure, sous réserve que le maître d'ouvrage soit en mesure d'évaluer la qualité des résultats. Ce dernier point est important. En effet, les résultats sont complexes à comprendre et à interpréter, et il apparaît nécessaire de pouvoir former la maîtrise d'ouvrage à leur analyse.

Toutefois, la réflexion sur la manière d'intégrer l'ACV au processus de décision d'un projet d'aménagement ne nous semble pas totalement aboutie. Le risque d'aujourd'hui est de n'utiliser l'ACV que pour effectuer une évaluation environnementale d'un projet. II y a donc une nécessité d'étudier plus en détails les processus de prise de décision dans un projet urbain, afin de cibler précisément les étapes auxquelles un besoin d'aide à la décision multicritères se fait réellement sentir, sur les performances environnementales du projet notamment, et les solutions qui pourraient être apportées par les logiciels tels que ARIADNE.

L'expérimentation précédente des logiciels d'ACV sur le projet d'aménagement à Lyon Confluence a déjà amené la collectivité à préciser ses objectifs environnementaux sur les nouveaux projets qu'elle entreprend. L'ACV lui a permis de cibler les indicateurs de performance environnementale qui, pour elle, sont prioritaires, et d'en fixer les niveaux de performance à atteindre. II serait donc intéressant pour Paris d'échanger avec cette collectivité sur cette 
expérience pour définir les indicateurs environnementaux et leur niveau d'exigence pour le périmètre parisien. À terme, un observatoire des bonnes pratiques serait également souhaitable.

\section{REMERCIEMENTS}

Nous remercions Benoit de Saint Martin (Direction de l'Urbanisme de la Ville de Paris), Anne Makovsky (SEMAVIP), Rémi Ferrand (Dussapin, Leclercq, architectes urbanistes) qui nous ont fourni un grand nombre d'information sur le projet de la ZAC Claude-Bernard, ses objectifs et son évolution, ainsi que les architectes qui ont pris le temps de nous préciser les caractéristiques environnementales des bâtiments en cours de construction.

\section{BIBLIOGRAPHIE}

CAP Terre (2006). Assistance à Maîtrise d'Ouvrage pour la Qualité Environnementale de la ZAC Claude Bernard - Quai de la Charente - canal Saint-Denis, Paris Nord-Est/l gème: Étude d'aide a la décision énergétique, Cap Terre, 20 P.

Chevalier, J. (2009). «Analyse du cycle de vie, utilisation dans le secteur de la construction », Techniques de l'ingénieur, NoG5 880.

Cole, R. CoOPER, I. KoHler, N, LÜTZKendorf et T. SMith, P. (1992). Buildings and the Environment. Proceedings of the International Research Workshop, Cambridge, September 1992.

FrischineCht, R., N. Jungbluth, H.-J. Althaus, G. DOKA, T. Heck, S. Hellweg, R. Hischier, T. NemeCek, G. Rebitzer, et M. SPIELMANN (2004). Overview and Methodology, Ecoinvent Report No. I, Swiss Centre for Life Cycle Inventories, Dübendorf, Suisse, 75 p.

GOBIN, CHR. (à paraître). «L'écoconception en construction », Technique de l'ingénieur.

OLIVA, J-P. (2006). L'isolation écologique, conception, matériaux et mise en œuvre, Terre Vivante, 238 p.

Peuportier, B. et I. Blanc Sommereux (1990). «Simulation tool with its expert interface for the thermal design of multizone buildings », International Journal of Solar Energy, vol. 8, p 109-120.

Peuportier, B., E. Popovici et M. Trocme (2006). Analyse de cycle de vie à l'échelle du quartier, Séminaire ADEQUA Quartiers Durables, Chambéry, octobre 2006.

Peuportier, B., D. Kellenberger, D. Anink, H. Mötzl, A. ANDerson, S. VARes, J. CheValier et H. KöNIG (2004). Inter-comparison and benchmarking of LCA-based environmental assessment and design tools ", Sustainable Building 2004 Conference, Warsaw, paper $\mathrm{n}^{\circ} 75,5 \mathrm{p}$.
Peuportier, B. Kohler et N. Boonstra, C. (1997). «European project REGENER, life cycle analysis of buildings ». 2nd International Conference "Buildings and the environment », Paris, Juin 1997.

Polster, B. (1995). Contribution à l'étude de l'impact environnemental des bâtiments par analyse du cycle de vie, thèse de doctorat, Paris, École des Mines de Paris, 203 p.

Popovicl, E. (2006). Contribution à l'analyse de cycle de vie des quartiers, thèse de doctorat, Paris, École des Mines de Paris, 209 p.

Salomon, T., R. Mikolasek et B. Peuportier (2005). « Outil de simulation thermique du bâtiment, COMFIE », Journée SFT-IBPSA «Outils de simulation thermo-aéraulique du bâtiment », La Rochelle, 8 p.

Tardieu, C., About-De Chastenet, C., Colombert, M., Diab, Y., Gobin, C., Herfray, G., Jarrin, T., Peuportier, B. et M. TROCME (20II). « Analyse de cycle de vie et aide à la décision : cas des bâtiments parisiens ». Colloque "La modélisation de la ville: du modèle au projet urbain ॥, École des Ponts ParisTech, Marne la Vallée, 23 et 24 février 2011. 